\title{
Ausschreibung: Publizistenpreis der deutschen Bibliotheken 2020
}

http://doi.org/10.1515/bd-2020-0018

Die bibliothekarischen Verbände Berufsverband Bibliothek Information e.V. (BIB), der Verein Deutscher Bibliothekarinnen und Bibliothekare e.V. (VDB) und der Deutsche Bibliotheksverband e.V. (dbv) schreiben gemeinsam den Publizistenpreis der deutschen Bibliotheken („Helmut-Sontag-Preis“) aus. Der Preis wird jährlich verliehen. Das Preisgeld beträgt 7.500 Euro.

Dieser Publizistenpreis zeichnet Journalist`innen oder Redaktionsteams aller Medien aus, die ein zeitgemäßes Bild von Bibliotheken, ihrem Umfeld und den sie beeinflussenden Entwicklungen vermitteln. Erwartet werden Beiträge, die solide recherchiert sind, nachhaltig die Thematik bearbeiten und sich mit ihr differenziert auseinandersetzen.

Wir bitten um begründete Vorschläge zusammen mit einer aussagekräftigen Dokumentation der seit 2018 veröffentlichten, einschlägigen Publikationen. Auch Eigenbewerbungen sind möglich. Über die Vergabe des Preises entscheidet eine unabhängige Jury. Der gleiche Beitrag kann nicht ein weiteres Mal eingereicht werden. Weiterhin darf es sich nicht um eine Auftragsarbeit handeln. Der Preis wird am 29. Mai 2020 im Rahmen der Abschlussveranstaltung des Bibliothekartages in Hannover verliehen.

Vorschläge können bis zum 28.02.2020 (Einsendeschluss) - bevorzugt in elektronischer Form - gesendet werden an:

Deutscher Bibliotheksverband e.V. (dbv)

„Publizistenpreis 2020“

Fritschestr. 27-28

10585 Berlin

E-Mail: baessler@bibliotheksverband.de

URL: www.bibliotheksverband.de

Der Preis geht auf eine Initiative des ehemaligen dbv-Vorsitzenden Helmut Sontag zurück und wird seit 1987 verliehen.

Ein Vorschlagsformular steht unter http://www.publizistenpreis.de zum Download zur Verfügung. Weitere Informationen über den Publizistenpreis können dort nachgelesen oder von der dbv-Bundesgeschäftsstelle angefordert werden. 


\section{Kontakt:}

Kristin Bäßler, Leitung Kommunikation

Tel.: 030 / 644989925 oder E-Mail: baessler@bibliotheksverband.de 